\title{
The "funitel" technique for endoscopic target biopsy at a biliary bifurcation
}

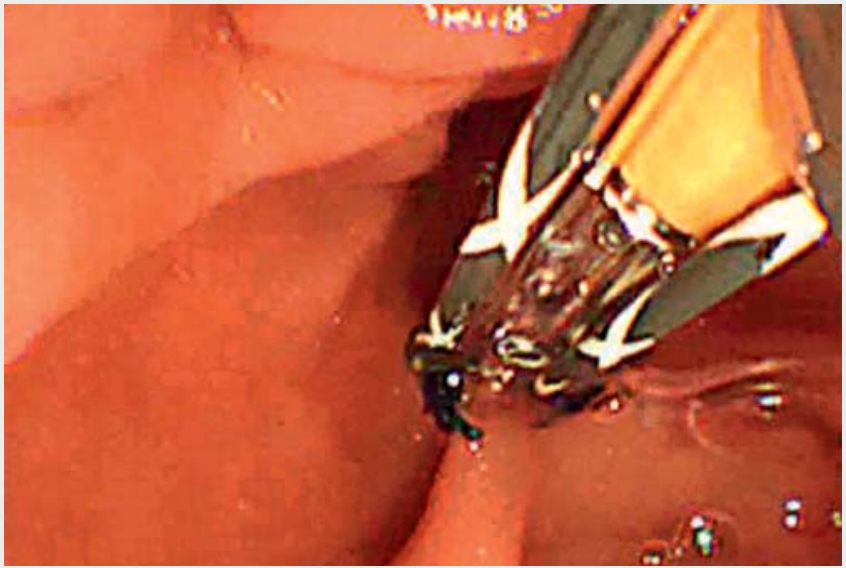

Video 1 The "funitel" technique for target biopsy at a biliary bifurcation during endoscopic retrograde cholangiopancreatography.

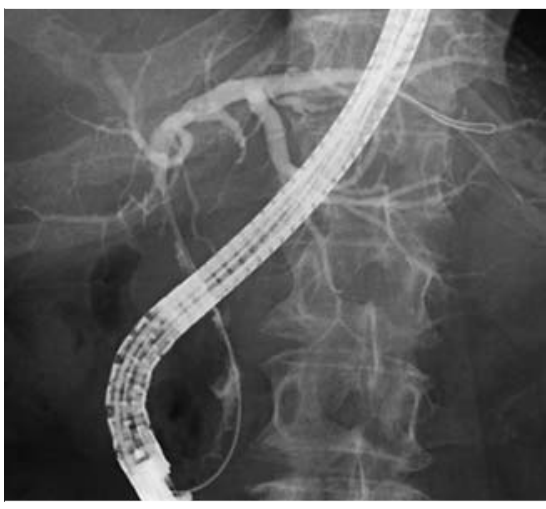

- Fig. 1 Cholangiogram delineating a stricture between the right hepatic duct and hilar bile duct with irregularity of the distal bile duct.
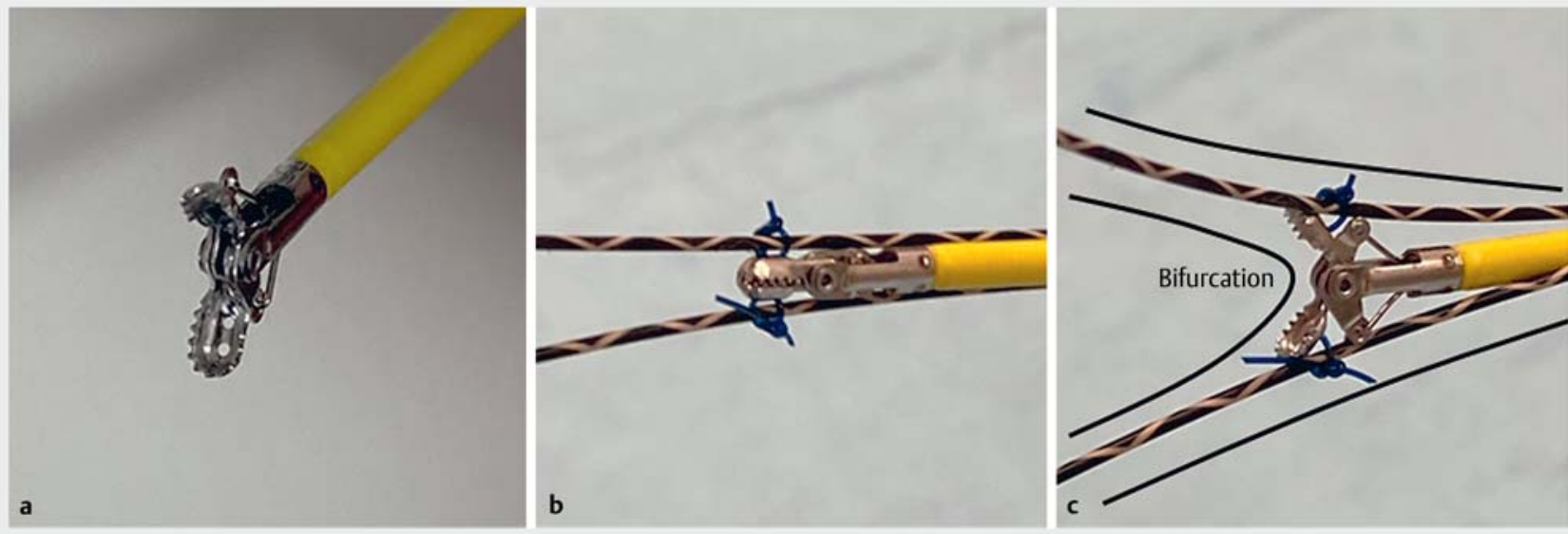

- Fig. 2 The "funitel" technique for targeted biopsy of biliary epithelium at the bifurcation of B2 and B3 during endoscopic retrograde cholangiopancreatography. a Photograph of biopsy forceps (Radial Jaw 4 pediatric; Boston Scientific, Marlborough, Massachusetts, USA) with two holes at each cup, which are designed to facilitate tissue acquisition. $\mathbf{b}$ A looped nylon thread passed through the holes of each cup of the forceps, which allows the forceps to be advanced over prepositioned guidewires. c Schema of the two guidewires and positioning of the biopsy forceps at the bifurcation.

Endoscopic retrograde cholangiopancreatography (ERCP) with biopsy is a gold-standard procedure for pathological assessment of spreading of cholangiocarcinoma $[1,2]$. Tumor existence at landmark biliary bifurcations should be assessed to consider the indication and extent of surgical resection. However, technical difficulties in pushing biopsy forceps against a biliary bifurcation may inhibit precise target biopsy. Here, we present a simple modification of widely used biopsy forceps for highly selective biopsy at the bifurcation of bile ducts at segments II and III (B2 and B3) ( $\vee$ Video 1$)$.
An 80-year-old woman with suspected hilar cholangiocarcinoma was referred to our centre for hepatopancreatoduodenectomy ( $\triangleright$ Fig. 1 ). We conducted ERCP and obtained tissue specimens from hilar and distal bile ducts, all of which revealed adenocarcinoma. Tumor absence at the bifurcation of B2 and B3 was a prerequi- 


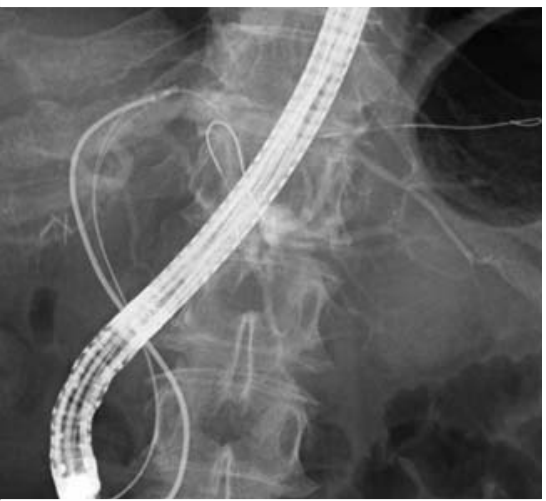

- Fig. 3 Biopsy forceps grasping biliary epithelium at the bifurcation of B2 and B3.

site for curative resection. During the following session, no obvious cancerous epithelium at the bifurcation could be visualized using digital cholangioscopy (SpyGlass DS; Boston Scientific, Marlborough, Massachusetts, USA), although the visualization was not good. Cholangioscopy-guided biopsy for pathological confirmation acquired only a small amount of tissue. Therefore, we added a looped nylon thread to each cup of the standard biopsy forceps (Radial Jaw 4 pediatric; Boston Scientific) and inserted the forceps with loops over 0.025-inch guidewires (VisiGlide 2; Olympus, Tokyo, Japan) positioned in B2 and B3 (> Fig. 2). The forceps were successfully pushed against the bifurcation, and enough tissue was obtained (> Fig.3). Based on positive pathological findings, the disease was considered unresectable, and chemotherapy was administered.

High costs and the limited amount of tissue obtained via mini biopsy forceps have been drawbacks of peroral cholangioscopy with biliary biopsy [3-5]. The "funitel" technique presented here would help to target lesions at biliary bifurcations at minimal additional cost. Biopsy forceps with holes in the cups and smooth guidewires may facilitate this technique.

Endoscopy_UCTN_Code_TTT_1AR_2AD
Competing interests

Dr. Nakai declares research funding from Boston Scientific Japan, Century Medical, Fujifilm, Gadelius Medical, Hitachi Medical, Kaneka, and Medico's Hirata. The current work was not supported by any of these companies. The remaining authors declare that they have no conflict of interest related to this article.

The authors

Tsuyoshi Hamada' ${ }^{\complement}$, Tomotaka Saito', Kensaku Noguchi ${ }^{1}$, Yousuke Nakai ${ }^{1}, 2$, Mitsuhiro Fujishiro ${ }^{1}$

1 Department of Gastroenterology, Graduate School of Medicine, The University of Tokyo, Tokyo, Japan

2 Department of Endoscopy and Endoscopic Surgery, The University of Tokyo Hospital, Tokyo, Japan

\section{Corresponding author}

\section{Yousuke Nakai, MD, PhD}

Department of Endoscopy and Endoscopic Surgery, The University of Tokyo Hospital, 7-3-1 Hongo, Bunkyo City, Tokyo 113-8655, Japan

ynakai-tky@umin.ac.jp

\section{References}

[1] Nakai $\mathrm{Y}$, Isayama H, Wang HP et al. International consensus statements for endoscopic management of distal biliary stricture. J Gastroenterol Hepatol 2020; 35: 967-979

[2] Sun B, Moon JH, Cai Q et al. Asia-Pacific consensus recommendations on endoscopic tissue acquisition for biliary strictures. Aliment Pharmacol Ther 2018; 48: 138-151

[3] Minami H, Mukai S, Sofuni A et al. Clinical outcomes of digital cholangioscopy-guided procedures for the diagnosis of biliary strictures and treatment of difficult bile duct stones: a single-center large cohort study. J Clin Med 2021; 10: 1638

[4] Kulpatcharapong S, Pittayanon R, Kerr S] et al. Diagnostic performance of different cholangioscopes in patients with biliary strictures: a systematic review. Endoscopy 2020; 52: 174-185
[5] de Vries AB, van der Heide F, Ter Steege RWF et al. Limited diagnostic accuracy and clinical impact of single-operator peroral cholangioscopy for indeterminate biliary strictures. Endoscopy 2020; 52: 107-114

Bibliography

Endoscopy 2022; 54: E637-E638

DOI 10.1055/a-1730-4410

ISSN 0013-726X

published online 4.2.2022

(C) 2022. Thieme. All rights reserved. Georg Thieme Verlag KG, Rüdigerstraße 14, 70469 Stuttgart, Germany

\section{ENDOSCOPY E-VIDEOS}

https://eref.thieme.de/e-videos

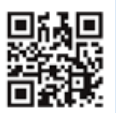

Endoscopy E-Videos is an open access online section, reporting on interesting cases and new techniques in gastroenterological endoscopy. All papers include a high quality video and all contributions are freely accessible online. Processing charges apply (currently EUR 375), discounts and wavers acc. to HINARI are available.

This section has its own submission website at

https://mc.manuscriptcentral.com/e-videos 